\title{
Measuring the Electron's Charge and the Fine-Structure Constant by Counting Electrons on a Capacitor
}

\section{E. R. Williams and} Ruby N. Ghosh,

National Institute of Standards and Technology, Gaithersburg, MD 20899

\section{and}

\section{John M. Martinis}

National Institute of Standards and Technology, Boulder, CO 80303
The charge of the electron can be determined by simply placing a known number of electrons on one electrode of a capacitor and measuring the voltage, $V_{s}$, across the capacitor. If $V_{s}$ is measured in terms of the Josephson volt and the capacitor is measured in SI units then the fine-structure constant is the quantity determined. Recent developments involving single electron tunneling, SET, have shown how to count the electrons as well as how to make an electrometer with sufficient sensitivity to measure the charge.

Key words: calculable capacitor; coulomb blockade; electron charge; electron counting; fine-structure constant; single electron tunneling.

Accepted: February 3, 1992

\section{Introduction}

The recent development of single-electron devices $[1,2,3,4,5]$ has made possible a new and very precise technique to measure the charge of an electron. These devices are based on metal/insulator/ metal tunnel junctions whose current-voltage $(I-V)$ characteristics are determined by individual electron tunneling events. If such a device is used to place $n$ electrons on a capacitor of capacitance $C_{s}$ and the resulting voltage $V_{\mathrm{s}}$ is measured, the electron charge $e$ can be determined in terms of this voltage and capacitance, $n e=V_{\mathrm{s}} C_{\mathrm{s}}$. In practice, $V_{\mathrm{s}}$ is most precisely determined in terms of the voltage generated by a Josephson junction and thus the experiment will not be a determination of $e$ in SI units, but a measurement of $e$ in terms of the Josephson volt [6]. In this paper we show that such a measurement will soon be possible and that an accurate result would make a useful contribution to the field of fundamental physical constants.

\subsection{Relating the Fine-Structure Constant and the Electric Charge}

First, we address how this measurement relates to the fine-structure constant, $\alpha$. By relating the measured voltage to a Josephson voltage and the capacitance to SI capacitance as measured in a calculable capacitor experiment [7], we can calculate the fine-structure constant from the following simple equations:

$$
V_{\mathrm{s}}=\frac{n e}{C_{\mathrm{s}}}=j f_{j} \frac{h}{2 e}
$$

where $f_{j}$ is the frequency producing the Josephson steps and $j$ is an integer associated with the Josephson effect. (Note that in a Josephson array $j$ is the sum of all the integer steps of each junction.) Solving Eq. (1) for $e^{2} / h, \alpha$ is given by

$$
\alpha=\frac{\mu_{0} c e^{2}}{2 h}=\frac{j \mu_{0} c f_{j} C_{s}}{4 n}
$$


where $\mu_{0}$ is the permeability of vacuum and $C_{s}$ must be measured in SI units. Specifically, the capacitance must be related to the calculable capacitor experiment. In a calculable capacitor experiment a change of capacitance of $0.5 \mathrm{pF}$ can be measured in terms of the meter with an accuracy of $0.014 \mathrm{ppm}[7]\left(1 \mathrm{ppm}=1 \times 10^{-6}\right.$; throughout, all uncertainties are one standard deviation estimates). This capacitance can then be related with accuracies near the $0.01 \mathrm{ppm}$ level to a $10 \mathrm{pF}$ capacitor that is stable and transportable.

If this experiment to measure $e$ in terms of the Josephson volt and the calculable capacitor can be realized with high accuracy, it will provide a new path to $\alpha$. This new approach is similar to the measurement of $\alpha$ via the quantum Hall effect [8]. Although both experiments require a connection to the calculable capacitor, this new method is much more direct. The $0.5 \mathrm{pF}$ capacitance change used in most calculable capacitor experiments is a good match to the size of the capacitance that might be used to determine $e$; only one or two precision ratio transformer bridges need be involved in the calibration of $C_{5}$. By contrast, in the quantum Hall case a chain of calibrations might involve these intermediate standards: $0.5 \mathrm{pF}, 10 \mathrm{pF}, 100 \mathrm{pF}$, $1000 \mathrm{pF}, 100 \mathrm{k} \Omega, 10 \mathrm{k} \Omega, 1 \mathrm{k} \Omega$, and $6453.2 \Omega$. The most accurate value of $\alpha$ determined from the calculable capacitor and quantum Hall effect has an uncertainty of $0.024 \mathrm{ppm}[7,9]$ with $0.014 \mathrm{ppm}$ coming from the calculable capacitor and the rest from the chain of intermediate standards. An alternate determination of $\alpha$ using the proton gyromagnetic ratio in $\mathrm{H}_{2} \mathrm{O}$ and the quantum Hall effect but not the calculable capacitor, has an uncertainty of $0.037 \mathrm{ppm}$ [9]. However, these two results differ from each other by $(0.10 \pm 0.043) \mathrm{ppm}$. A value of $\alpha$ determined from the anomalous magnetic moment of the electron and quantum electrodynamic theory (QED) has an uncertainty of $0.007 \mathrm{ppm}$ [10] and its value lies between the two non-QED values. Therefore, the unexplained $0.10 \mathrm{ppm}$ difference in the non-QED values limits the accuracy to which QED theory is tested. Any measurement of $\alpha$ by this new SET method at the $0.1 \mathrm{ppm}$ level or better would be helpful in the field of fundamental constants [8].

\subsection{SET Devices}

K. K. Likharev and his colleagues [1] have long been proponents of the application of Coulomb blockade effects arising from the discreet charge of electrons to realize a precise current source. Their pioneering ideas and experiments paved the way for much of the recent progress. In the past few years, new devices employing metal/insulator/metal tunnel junctions have been demonstrated which could allow a precise measurement of the electron charge $e$ and thus $\alpha$. These include an electrometer [2], observation of single electron tunneling oscillations [3], a "turnstile" current source [4], and a "pump" current source [5]. A brief sketch of single-electron tunneling is presented to describe the operation of the electrometer and pump.

Consider a normal-metal tunnel junction biased by a current source and having a capacitance $C$. The change in energy of the junction after an electron tunnels through the barrier is $\Delta E=$ $e Q / C-e^{2} / 2 C$, where $Q$ is the charge across the junction and $e^{2} / 2 C$ is the Coulomb energy cost of the tunneling event. If the tunneling resistance of the junction is greater than the quantum unit of resistance, $R_{\mathrm{t}} \gg h / e^{2}$, and thermal fluctuations do not mask the charging energy, $k T \ll e^{2} / 2 C$, then a Coulomb blockade appears in the junction $I-V$ characteristic where the tunneling probability is greatly reduced for $|V|<e / 2 C$. At $50 \mathrm{mK}$, and using $1 \mathrm{fF}$ for the junction capacitance, one finds $(k T)$ / $\left(e^{2} / 2 C\right) \sim 1 / 20$, hence temperature effects are small but non-negligible. The single-electron devices exploit this Coulomb blockade of the tunneling current.

The Fulton-Dolan (SET) electrometer [2] is shown schematically in Fig. 1a. Aside from tunneling events, the electrode a between the two junctions and the gate capacitor $\mathrm{C}_{0}$ is an "island" electrically isolated from the circuit. The electrometer provides a very high impedance technique to measure the potential $U$. At a constant bias $V$, the device conductance as a function of gate voltage is periodic with the period $\Delta U=e / C_{0}$. The bias voltages $V$ and $U$ are chosen to maximize the sensitivity of the electrometer so that the device current is linearly proportional to small changes of the potential $U$. Such electrometers have demonstrated

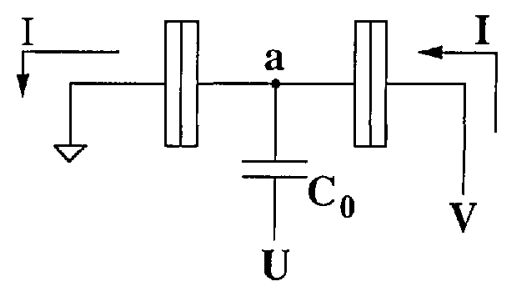

Fig. 1a. This circuit shows an electrometer formed by two tunnel junctions each having a capacitance $C$. The input is potential $U$ coupled to the isolated island a through $C_{0}$. 
charge sensitivity of $1.5 \times 10^{-4} e / \mathrm{Hz}^{1 / 2}$ at a frequency between 2 and $200 \mathrm{~Hz}$ [11].

Convincing experimental evidence of the SET oscillations producing the controlled transfer of electrons with the relation $I=e f$ was reported by Delsing et al. [3]. Geerligs et al. [4] were the first to demonstrate flat plateaus and observe a current with accuracies of better than $1 \%$ using the turnstile device. However, certain properties of the pump device suggest that it will be more precise in counting electrons and we therefore sketch its operation.

An electron pump [5] (see Fig. 1b) is a device similar to the electrometer, in which a single electron tunnels sequentially through the "islands" labeled $\mathbf{b}$ and $\mathbf{c}$. For a given voltage $V$, a controlled number of electrons can be transferred in either direction by appropriately cycling the gate voltages $U_{1}$ and $U_{2}$ at the frequency $f$. The pump also delivers an electron current at the rate $I=e f$. The direction of the current can be reversed simply by reversing the phase of $U_{1}$ and $U_{2}$. A pump based on four or five tunnel junctions operating at 1-10 $\mathrm{MHz}$ has been theoretically predicated to be able to deliver a known current with errors below the 1 ppm level $[12,13]$.

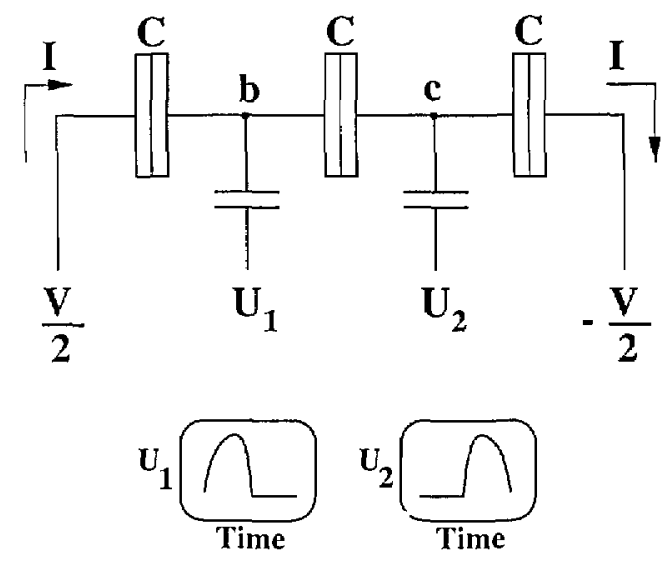

Fig. 1b. This circuit shows a three junction pump that pumps one electron per cycle of the wave-forms shown. The electron is first pumped to island b by potential $U_{1}$ then to $\mathrm{c}$ by $U_{2}$.

The currents produced by these pumps are only about $10^{-12} \mathrm{~A}$ and thus precision measurements will be a challenge. Direct measurement by sensing the voltage across a high resistance is a problem because it is difficult for metrologists to accurately calibrate the required high resistances. Capacitors, on the other hand, have already been accurately calibrated in a range that will be useful in the following experiment.

\section{Experimental Configurations [14]}

\subsection{Measurements of the Electric Charge $e$}

Figure 2a shows one possible configuration that incorporates these new SET devices to measure the electronic charge. A standard capacitor $C_{s}$ is connected to a current source (pump) and also to the input of a Fulton-Dolan electrometer via a coupling capacitor $\mathrm{C}_{c}$, forming an isolated island $\mathrm{a}$. Using the electrometer as a null detector a voltage $V_{s}$ is applied across $C_{s}$ to maintain the island $a$ at a fixed potential (near ground), while pumping electrons onto or off of the island. The stray capacitance to ground, $C_{\mathrm{g}}$, limits the sensitivity of the electrometer to $V_{\mathrm{s}}$. The voltage, $V_{\mathrm{s}}$, on the capacitor is adjusted so that the electrometer input is always at the same potential (near ground). Thus the current from the

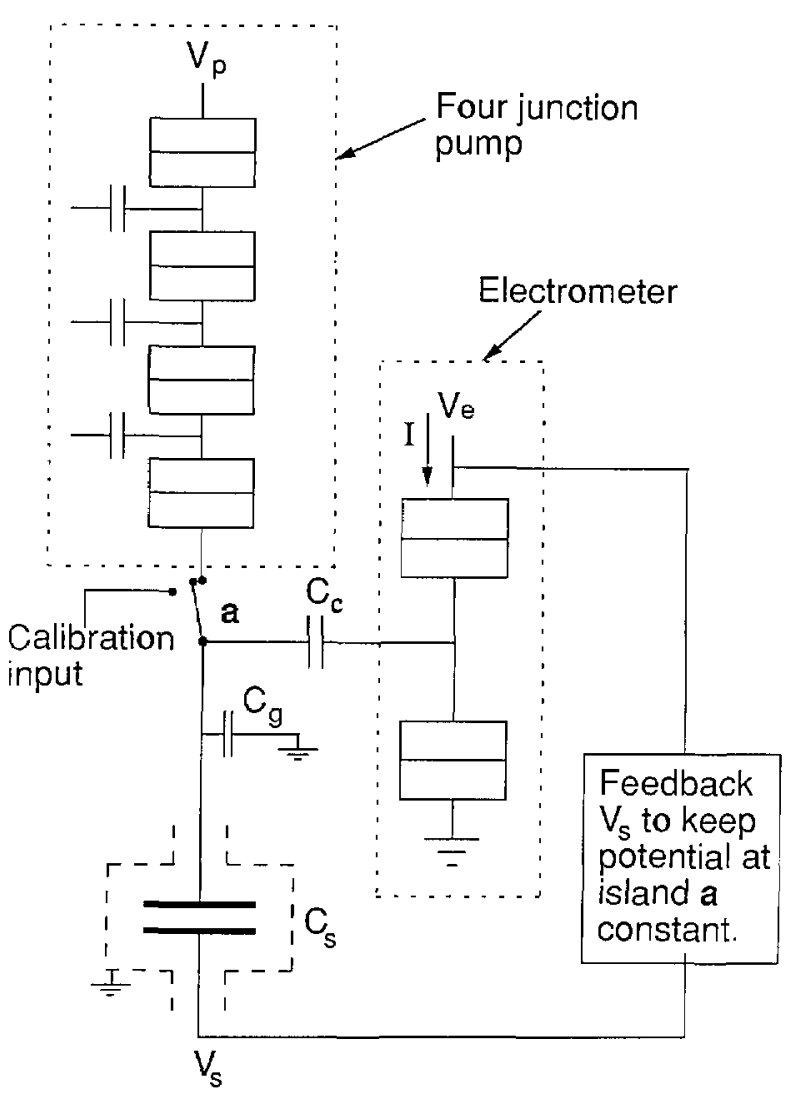

Fig. 2a. Proposed circuit for measuring $\alpha$. The voltage $V_{\mathrm{s}}$ required to keep the potential of the island a constant when $n$ electrons are pumped onto the island can be used to measure the electron charge in terms of $C_{\mathrm{s}}$ and $V_{\mathrm{s}}$. The electrometer monitors the potential at $\mathbf{a}$. 
pump serves as the charging current of the capacitor as the voltage is ramped up. A specific example is illustrated in Fig. 2b. With the pump operating at $6.2 \mathrm{MHz}$ for $10 \mathrm{~s}$, the voltage across a $1 \mathrm{pF}$ capacitor standard will charge to $10 \mathrm{~V}$ at a linear rate in order to keep the electrometer input constant. We stop the pump and measure the $10 \mathrm{~V}$ signal as well as any deviation of the electrometer from null. Reversing the pump for $20 \mathrm{~s}$ then brings the voltage to $-10 \mathrm{~V}$.

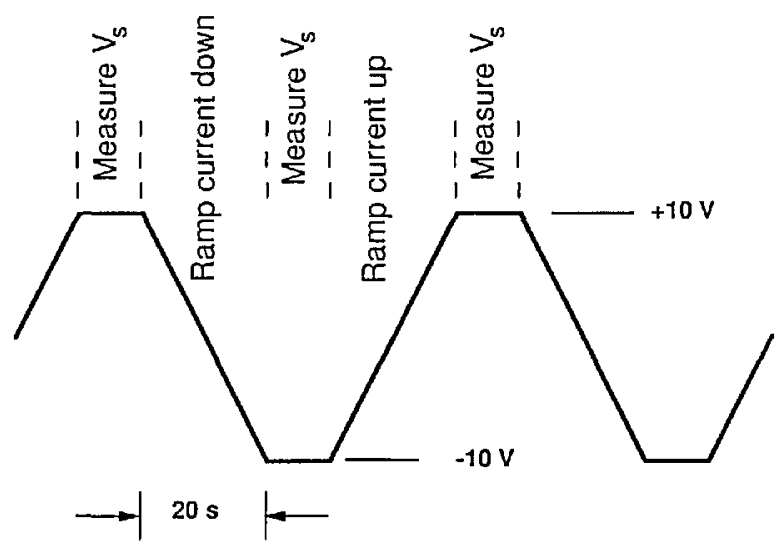

Fig. 2b. The measurement sequence for measuring $\alpha$, showing the potential $V_{\mathrm{s}}$ as a function of time.

In order to use a capacitor as a standard it must have a well defined capacitance; it thus is necessary to understand how precision capacitors are measured and under what circumstances they are well defined. Figure 3 shows the essential features of one side of a standard bridge that is used to compare capacitors $C_{1}$ and $C_{2}$. The dashed lines represent the grounded shield that prevents the potentials $V_{1}$ and $V_{2}$ from affecting the detector except through their respective capacitances. This shielding is critical to making precise capacitance comparisons. The bridge is balanced by adjusting the potentials $V_{1} / V_{2}=C_{2} / C_{1}, C_{g 1}$ and $C_{82}$ represent the stray capacitance to ground. The bridge balance is not affected by $C_{\mathrm{g} 1}$ because there is no voltage across $C_{g 1}$ when the bridge is balanced. However, $C_{g 1}$ does affect the detector sensitivity so it should be kept small. The capacitance $C_{\mathrm{g} 2}$ does not affect the balance as long as the impedance from the source to $C_{1}$ is small compared to the impedance of $C_{\mathrm{g} 2}$. Present day precision $10 \mathrm{pF}$ capacitance standards with $C_{\mathrm{g} 1}=200 \mathrm{pF}$ are compared at the $0.01 \mathrm{ppm}$ level at a frequency $\omega=10^{4}$ $\mathrm{s}^{-1}(1592 \mathrm{~Hz})$ using a room temperature detector with a noise figure of $20 \mathrm{e} / \mathrm{Hz}^{1 / 2}$. A SET electrometer should have similar noise performance at the same frequency and capacitance. Further improvements in the sensitivity of the SET electrometer are expected when measuring smaller capacitances.

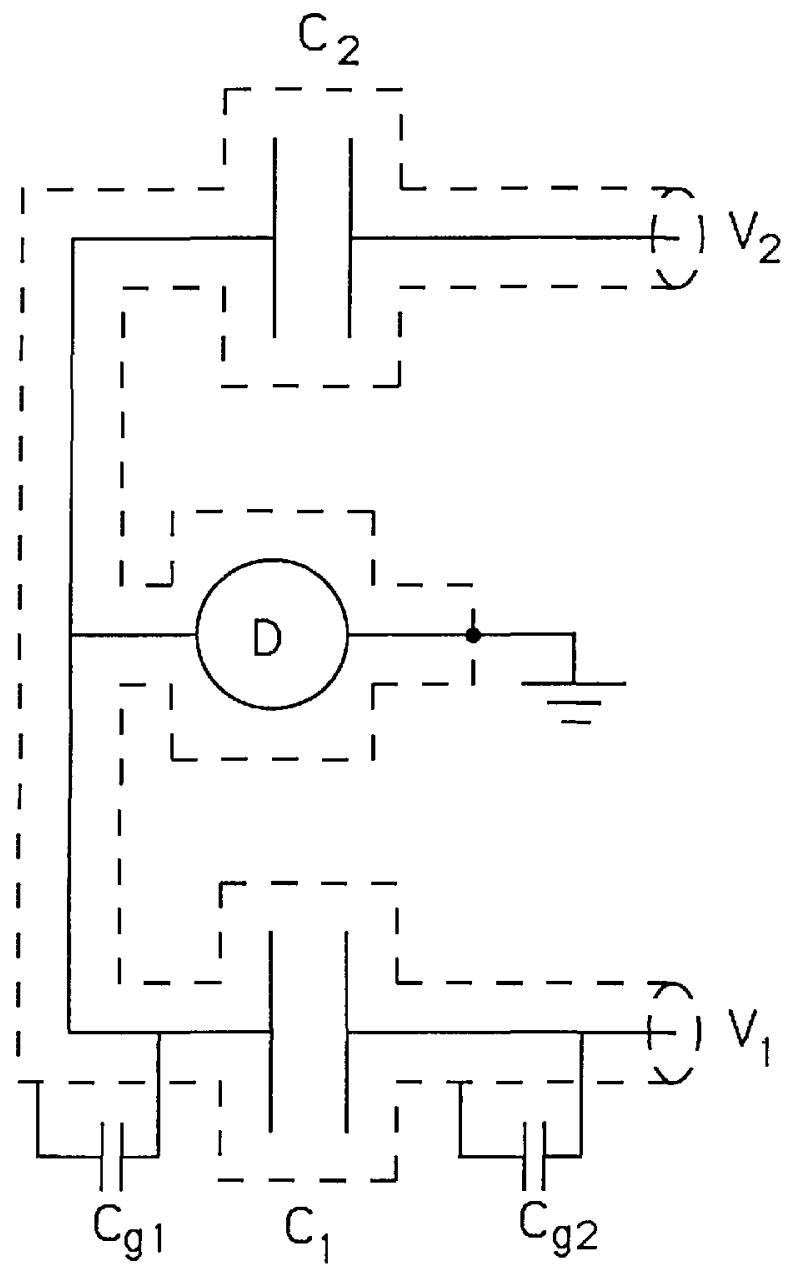

Fig. 3. Circuit showing half of a bridge used to compare capacitors $C_{1}$ and $C_{2}$. The potentials $V_{1}$ and $V_{2}$ are usually supplied by a precision transformer. The effect of stray capacitances $C_{\mathrm{z} 1}$ and $C_{\mathrm{g} 2}$ are discussed in the text.

The key to this experiment to measure $e$ using a capacitor is to replace the detector in Fig. 3 with a Fulton-Dolan electrometer as shown in Fig. 2a. By placing the electrometer at $20 \mathrm{mK}$ near the source of charge, we anticipate fewer problems from leakage and less stray capacitance to ground. In addition, we expect to gain from the impressive sensitivity of this device, reduce the leakage dramatically, and still make a well defined capacitor that can be calibrated in situ. 
Because of the requirement to shield the standard capacitor, it is easier initially to have this capacitor on a different chip from the electrometer and pump. As multilayer technology becomes incorporated into the junction fabrication process this capacitor could be placed on the same chip. Having it on a separate chip leads to some practical limits in the optimum choice for $\mathrm{C}_{\mathrm{s}}$. The sensitivity of the electrometer is approximately given by $C_{\mathrm{d}}\left(C_{\mathrm{s}}+C_{\mathrm{g}}\right)$, where $C_{\mathrm{g}}$ represents all other capacitances to ground. This two chip arrangement will increase $C_{\mathrm{g}}$, but we expect to keep it near $1 \mathrm{pF}$. Choosing $C_{\mathrm{s}}$ to be small both increases $V_{\mathrm{s}}$ and maximizes the electrometer sensitivity. A $1 \mathrm{pF}$ capacitance for $C_{s}$ constitutes a practical compromise.

Calibration of the capacitance requires making a connection to room temperature. However, the Fulton-Dolan electrometer could still be used by means of the calibration input switch shown in Fig. 2. Note that care must be taken to keep the capacitance from the island to ground low.

\subsection{Dual Pump Bridge}

Another interesting experiment (see Fig. 4) is to have two current sources (drawn as two, four-junction "pumps" in Fig. 4) feeding into an island a with a total capacitance of about $10 \mathrm{fF}$. The charge state of island $a$ is then measured with an electrometer. This geometry will not measure $e$ but will allow the very precise comparison of two current sources. Any difference in current from the two sources will show up as an accumulation of the island charge. The island capacitance is chosen to be large enough to greatly reduce the interaction of the two pumps but small enough to still have single charge resolution. Because the error is detected as an integrated charge on the island, only a short time is required to obtain extremely high precision. It would also be interesting to compare the error rate of a "pump" and a "turnstile."

\section{Future Prospects}

A new precision technique to measure the finestructure constant via Eqs. (1) and (2) has been described. At present SET experiments have been used to determine currents only at the 0.1 to $1.0 \%$ level, hence it is risky to predict the ultimate accuracy of the technique. Based on theoretical predictions $[12,13]$ we expect that the number of electrons could be measured with 1 ppm accuracy in a four junction pump. A five junction pump

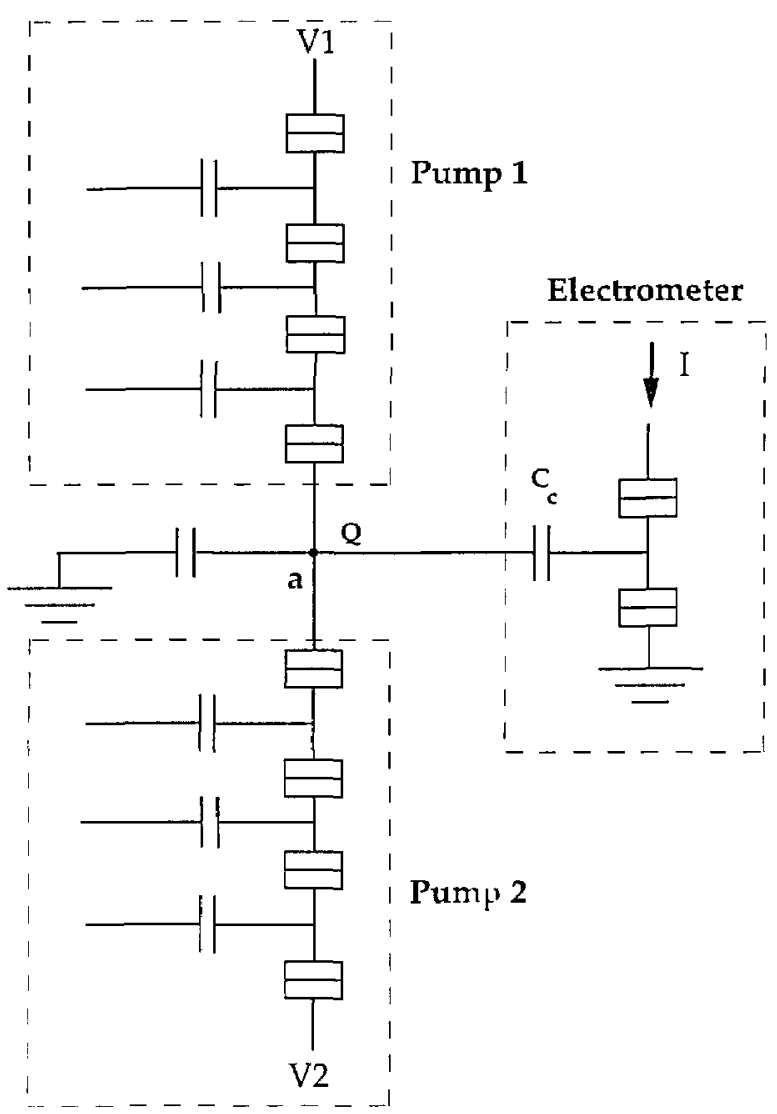

Fig. 4. A bridge circuit used to compare two pump circuits. The electrometer detects any accumulation of charge on island a.

should be more accurate still. At present the electrical standards needed to support this measurement do not appear to limit its accuracy. For example, capacitance standards of $10 \mathrm{pF}$ are compared between national laboratories near the 0.02 ppm level and $10 \mathrm{~V}$ Zener voltage references are also compared with $0.02 \mathrm{ppm}$ accuracy. In the first experiments these accuracies will likely not be reached, but much physics will be learned about SET devices as we make another accurate determination of $\alpha$.

\section{Acknowledgment}

We thank M. H. Devoret, D. Esteve, C. Urbina, H. Pothier, and P. Lafarge of Service de Physique du Solide et de Resonance Magnetique, Centre d'Etudes Nucleaires des Saclay, for many informative discussions about SET devices. We thank J. Q. Shields for crucial discussions about the techniques used to measure standard capacitors. Discussions with B. N. Taylor, Hans Jensen, and R. L. Kautz were also very useful. 


\section{References}

[1] D. V. Averin and K. K. Likharev, in Mesoseopic Phenomena in Solids, B. Al'tshuler, P. A. Lee, and R. A. Webb, eds., Elsevier, Amsterdam (1991) Chapter 6.

[2] T. A. Fulton and G. J. Dolan, Observation of SinglcElectron Charging Effccts in Small Tunnel Junctions, Phys. Rev. Lett. 59, 109-112 (1987).

[3] P. Delsing, K. K. Likharey, L. S. Kuzmin, and T. Claeson, Time-Correlated Singlc-Electron Tunneling in OneDimensional Arrays of Ultrasmall Tunnel Junctions, Phys. Rev. Lett. 63, 1861-1864 (1989) and Phys. Rev. B 42, 7439-7449 (1990).

[4] L. J. Geerligs, V. F. Anderegg, P. A. M. Holweg, J. E. Mooji, H. Pothier, D. Esteve, C. Urbina, and M. H. Devoret, Frequency-Locked Turnstile Device for Single Electrons, Phys.Rev. Lett. 64, 2691-2694 (1990).

[5] H. Pothier, P. Lafarge, C. Urbina, D. Esteve, and M. H. Devoret, Single-Electron Pump Based on Charging Effects, Europhys. Lett. 17, 249-254 (1992).

[6] Resolution 6 of the 18th Confèrence Gènèrale des Poids et Mesures. See B. N. Taylor, ed., The International System of Units (SI), Natl. Inst. Stand. Technol. Spec. Pub. 330 (1991) p. 45.

[7] J. Q. Shields, R. F. Dziuba, H. P. Layer, New Realization of the Ohm and Farad Using the NBS Calculable Capacitor, IEEE Trans. Instrum. Meas. 38, 249-251 (1989).

[8] B. N. Taylor and E. R. Cohen, How accurate are the Josephson and Quantum Hall Effects and QED?, Phys. Lett. A 153, 308 (1991).

[9] M. E. Cage, R. F. Dziuba, R. E. Elmquist, B. F. Field, G. R. Joncs, Jr., P. T. Olsen, W. D. Phillips, J. Q. Shields, R. L. Steiner, B. N. Taylor, and E. R. Williams, NBS Determination of the Fine-Structure Constant and the Quantizcd Hall Resistance and Josephson Frequency-toVoltage Quotient in SI Units, IEEE Trans. Instrum. Meas. 38, 284-289 (1989).

[10] T. Kinoshita and W. B. Lindquist, Eighth-order Magnetic Moment of the Electron. V. Diagrams Containing No Vacuum-Polarization Loop, Phys. Rev. D 42, 636-655 (1990).

[11] L. J. Geerligs, V. F. Anderegg, and J. E. Mooij, Tunneling Time and Offset Charging in Small Tunnel Junetions, Physica B165/166, 973-974 (1990).

[12] H. Pothier, 1991, Blocage de Coulomb et Transfert d'Electons Un Par Un, PhD Thesis, de l'Universite Paris 6.

[13] Hans Jansen and J. Martinis, private communications.

[14] The configurations shown here were first presented by the authors as a poster paper at the NATO Adyaneed Study Institute on Single Charge Tunneling, Les Houches, France, March 1991. J. Martinis will present a paper on this subject at the Conference on Precision Electromagnetic Measurements, June 1992, in Paris France.
About the authors: Edwin R. Williams is a physicist in the Electricity Division at NIST. Ruby N. Ghosh is an American Society of Engineering Education Postdoctoral Fellow in the Electricity Division at NIST. John M. Martinis is a physicist in the Electromagnetic Technology Division at NIST. The National Institute of Standards and Technology is an agency of the Technology Administration, U.S. Department of Commerce. 